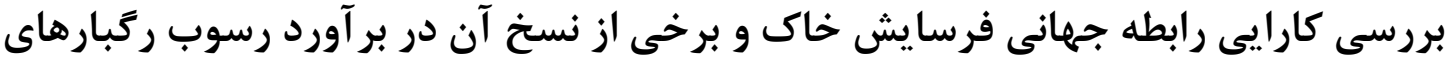

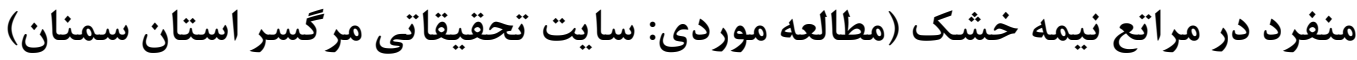

\author{
مجيد كار تر '، محمدرضا جوادى ‘، سيد على اصغر هاشمى "و مريم محمد ابراهيمى'

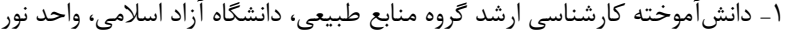

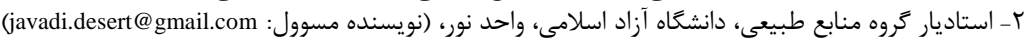

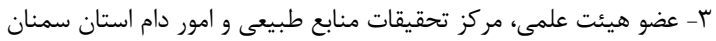

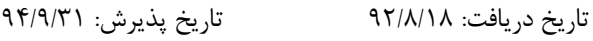

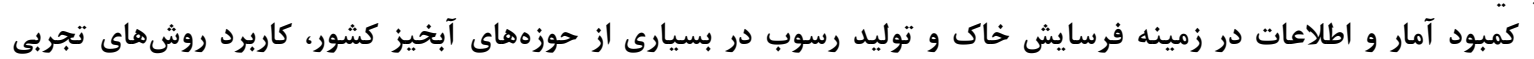

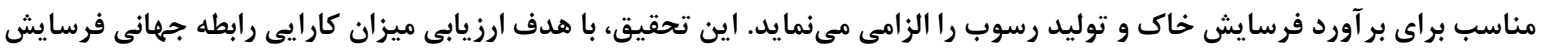

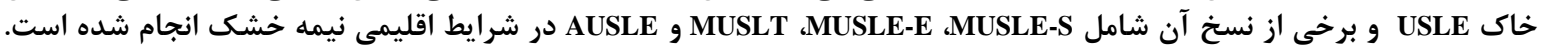

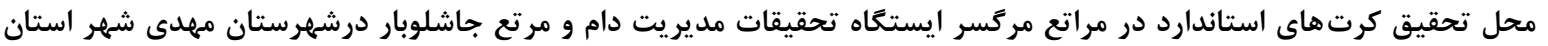

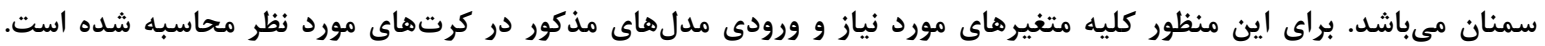

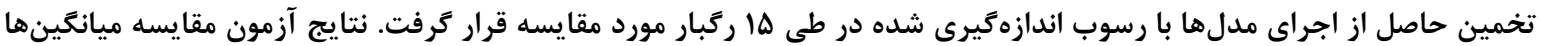

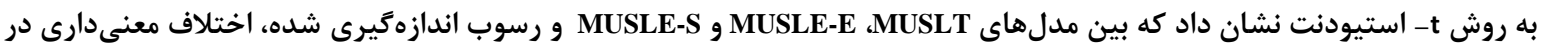

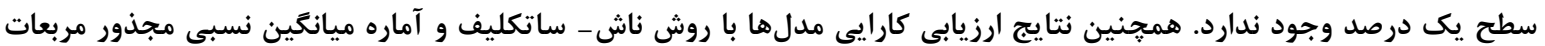

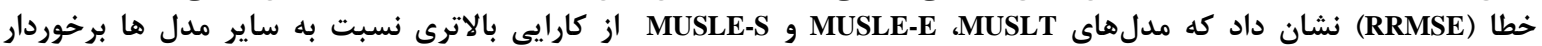

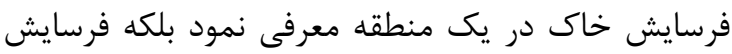

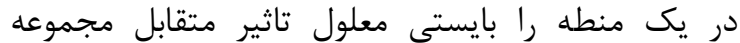

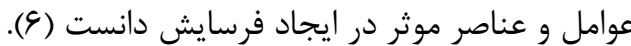

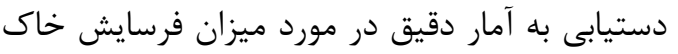

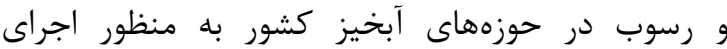

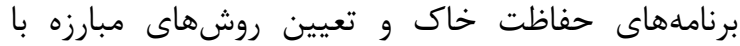

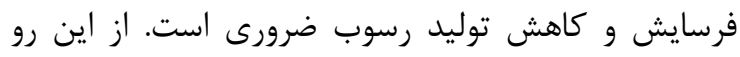

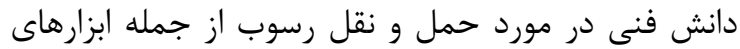

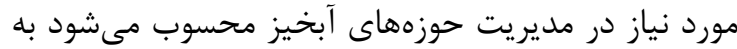

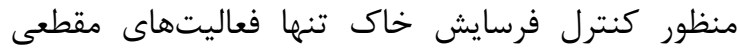

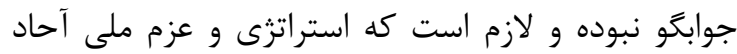

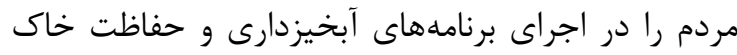

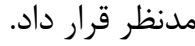

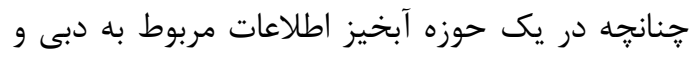

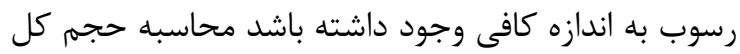

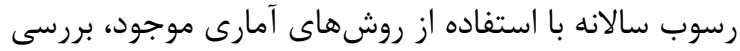

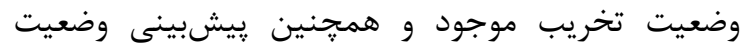

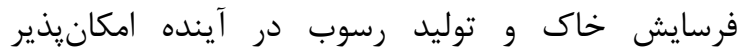
مىباشد. ولى عدم وجود و يا كمبود آمار و اطلاعات در آندان
مقدمه

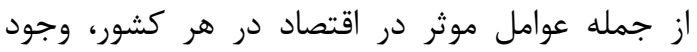

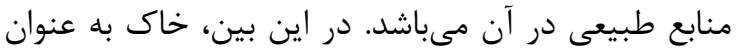

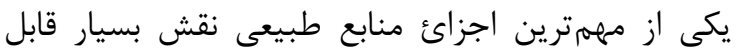

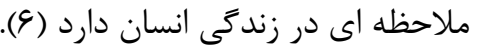
با تشديد بهرهبردارىهاى اندان انسان از طارد طبيعت، فرسايش

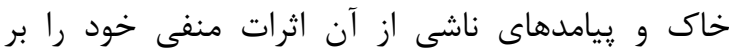

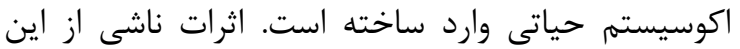

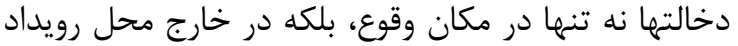

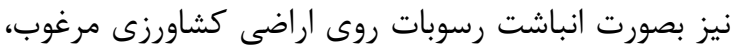

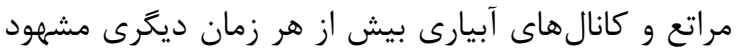
و نمايان است (ب) (1).

امروزه فرسايش خاك و توليد رسوب مشكل عمده اي إي

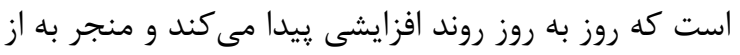

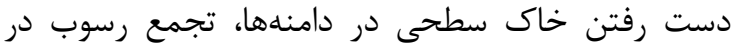

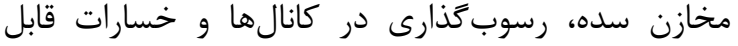

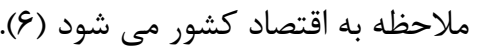

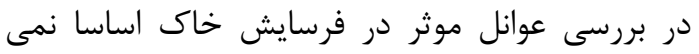

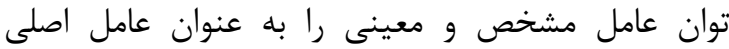




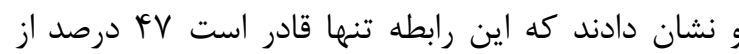

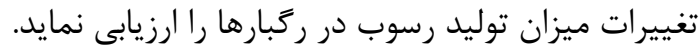

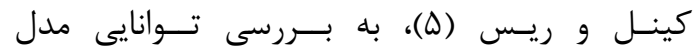
USLE Rocky Creek

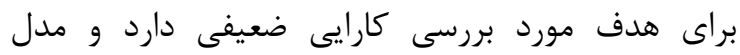
تبا كارايى بيشتر نسبت به به مدل USLE-M

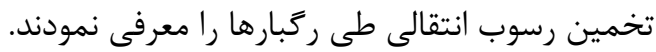

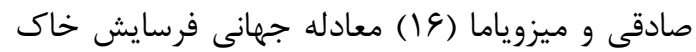

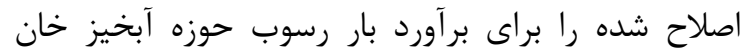

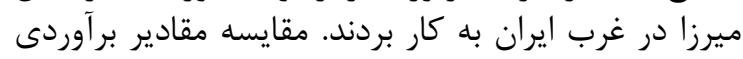

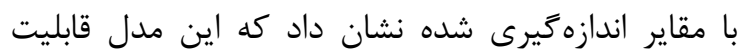

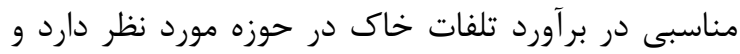

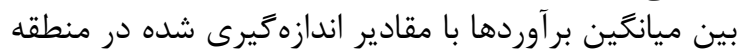

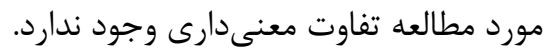

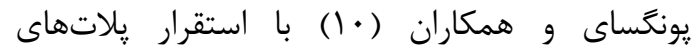

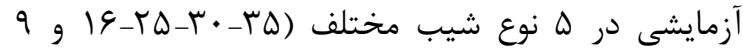
درصد) و اندازهيرى و ثبت رسو رسوب حاصل از نمونه ركبار

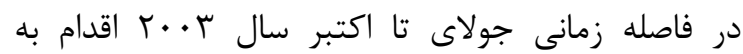
ارزيابى و واسنجى معادله جهانى فرسايش نموده و و بيان إنى

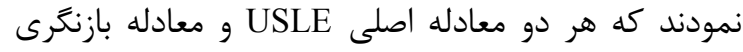

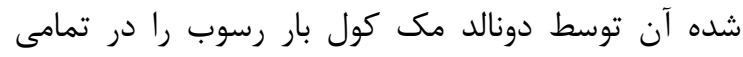
شيبها بيشتر از مقدار واقعى برآورد مى نمايند.

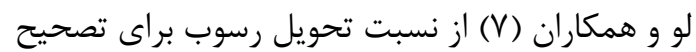
اثر كاهش باررسوبى در خروجى استفاده نمودند. نتايح

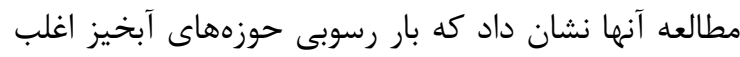

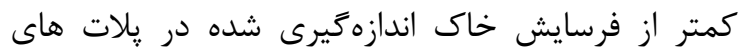

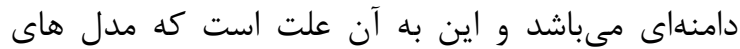

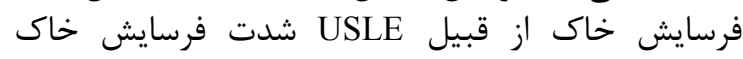

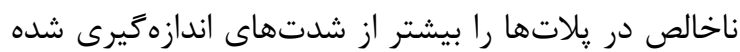

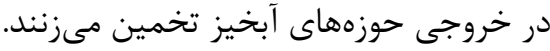

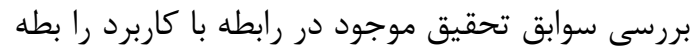

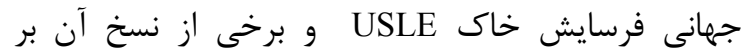

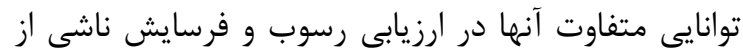

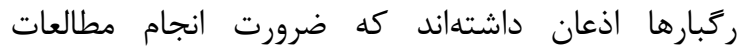
كستردهتر در اين زمينه رار ران أناكيد مى كند.

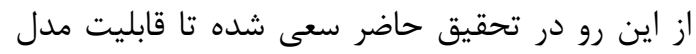

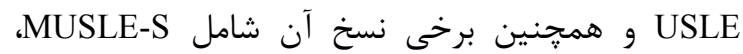
منSLST ،MUSLE-E منطقه سفيد دشت در شهرستان سمنان به دان دليل

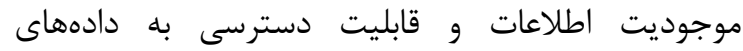
اندازهزيرى شده مورد مقايسه و ارزيابى قرار گيرد.
زمينه فرسايش خاك و توليد رسوب در بسيارى از

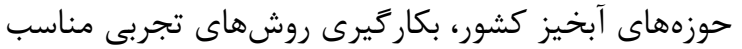

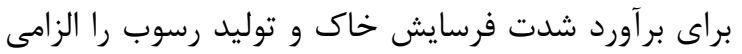

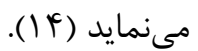

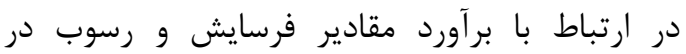

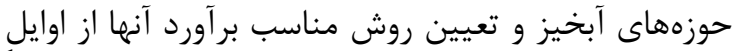

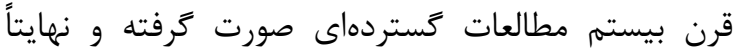
منجر به تهيه رابطه جهانى فرسايش خاك لهات كه به طور كسترده در سرتاسر جهان براى تخاى تخمين سالانه

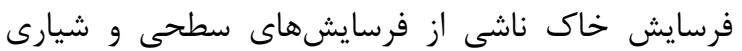

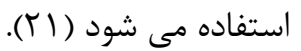

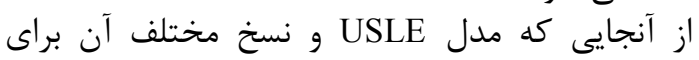

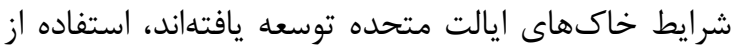

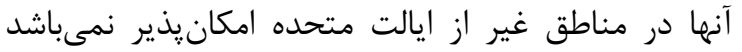

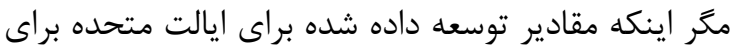

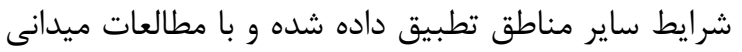
تاييد گردد (צ). غلامى (Y)، در حوزه آبخيز سد قشادلاق در در استان

كردستان به بررسى قابليت مدلهاى (MUSLE-E TAM و USLE ،AUSLE ،MUSLE-S ،MUSLT يرداخته و نتيجه گرفت كه تنها مدلهاى MUSLE-S و TAM منطقه مورد مطالعه دارا مي بـاشند.

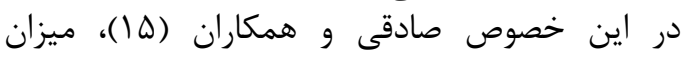

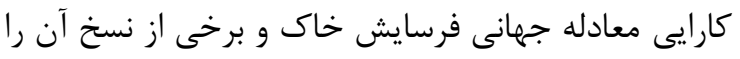

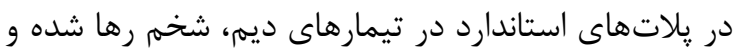

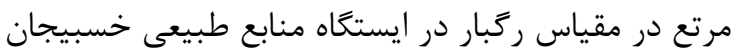

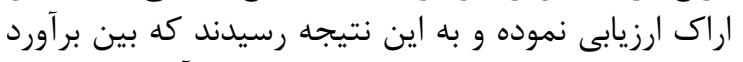

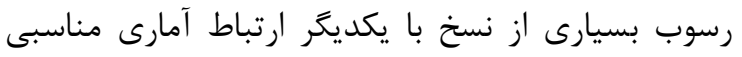

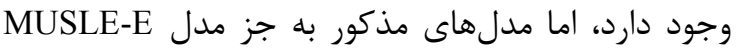

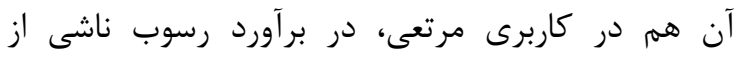
ركبارها در تيمارهاى مورد مقايسه كارايى نداشتهاند.

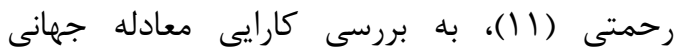

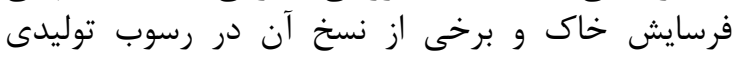

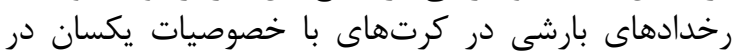

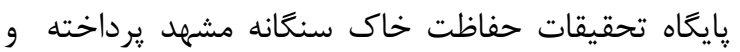
نتيجه گرفت كه مقادير برآوردى جهار روش حفاطن MUSLT ، MUSLE-E معنى دارى با رسوب مشاهداتى داشته و مىتوانند روند تغييرات رسوب ناشى از ركبار را به خوبى بـ برآ رآورد نمايند.

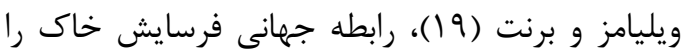
براى تخمين ميزان رسوب رگبار در آمريكا استفاده نموده 
مورد مطالعه در زون البرز جنوبى قرار داشته و متاثر از مواد و روشها

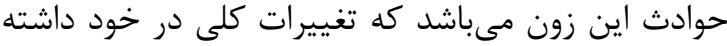

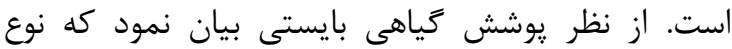

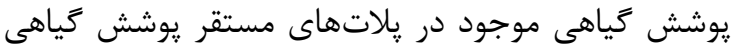

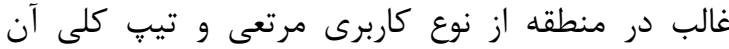
بالشتكى- تراس مى مباشد. خاك هاى منطقه موجود در محرى محل استقرار ريلاتها از

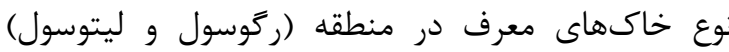

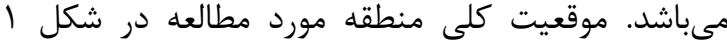

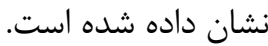

تحقيق حاضر در سايت تحقيقات مرگسر با مختصات

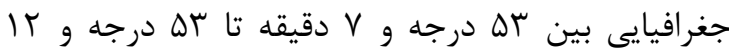

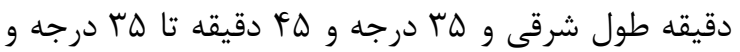

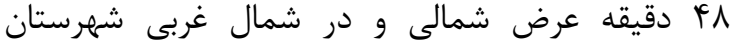

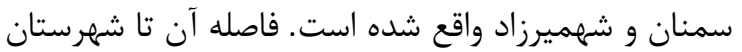

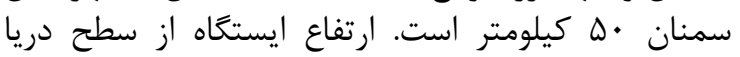
تو FV..

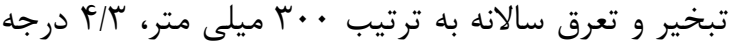

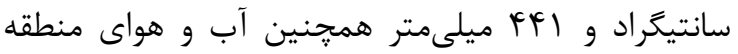

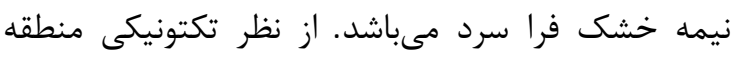

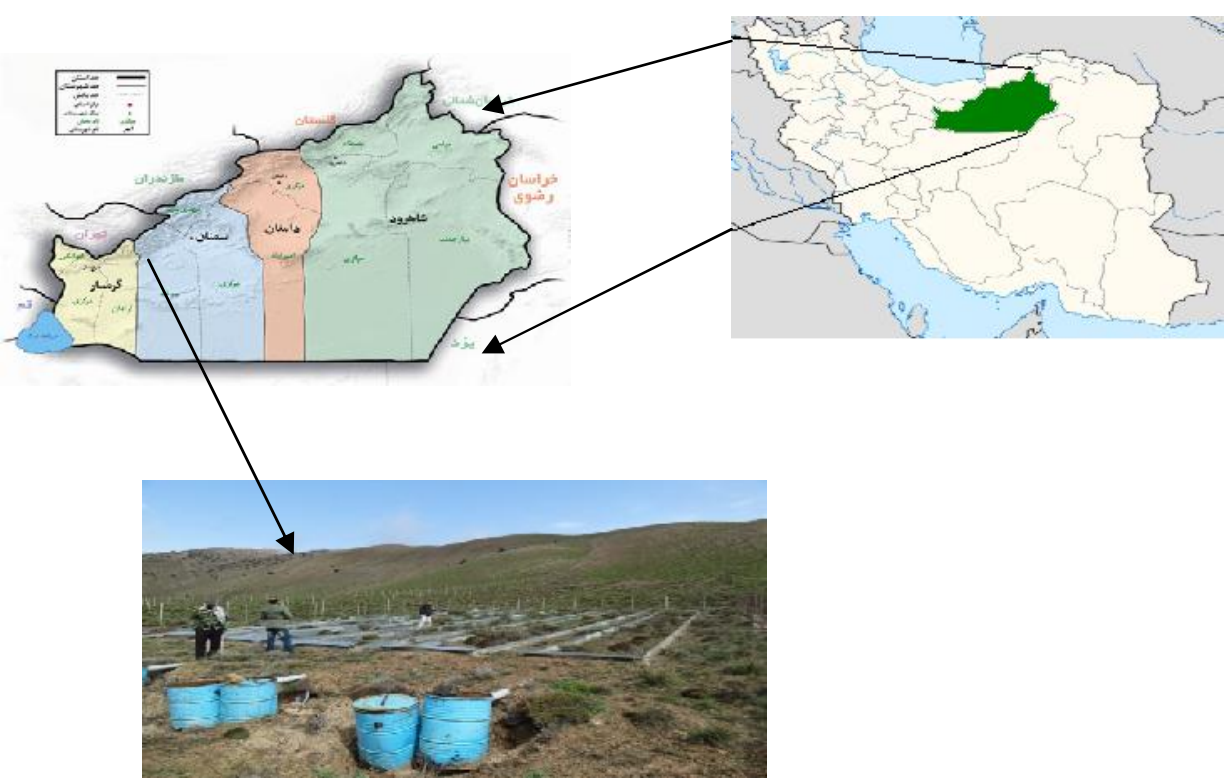

شكل I - موقعيت منطقه مورد مطالعه و شماى كلى يلاتها.

نمونههاى رواناب و رسوب جمعآورى شده به آزمايشگاه

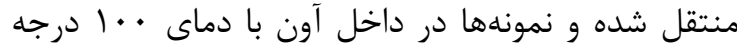

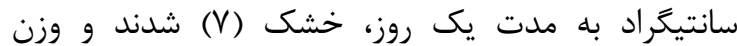

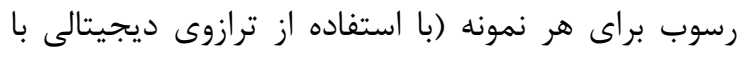

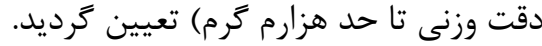
در اين سايت طى سالهاى

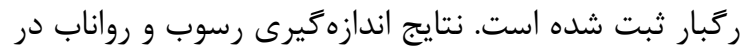

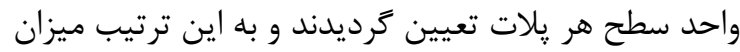
رسوبدهى هر يلات براى هر رخبار تعيين شدند.
در اين تحقيق از يلاتهاى موجود در ايستگاه

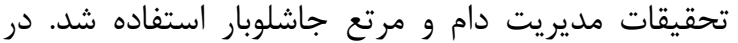

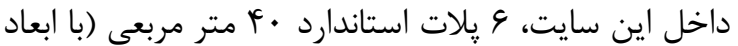

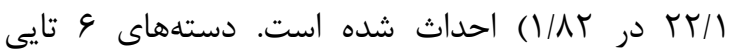

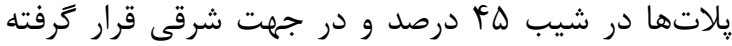

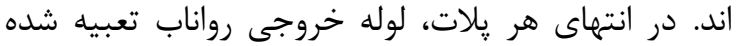

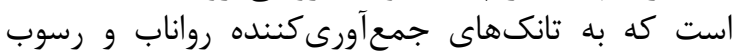

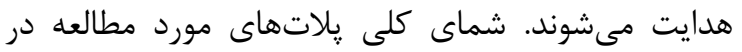

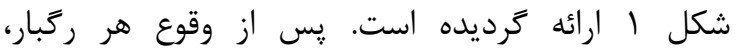



بررسى كارايى رابطه جهانى فرسايش خاك و برخى از نسخ آن در برآورد رسوب ركبارهاى منفرد در.

جدول ا- مشخصات رَّبارهاى انتخابى مدلهاى مورد مطالعه و رسوب توليدى ناشى از آنها

\begin{tabular}{|c|c|c|c|c|c|}
\hline ر رسوب مشاهدهاى & (مترم رواناب) & (سانتى متر برسم ساعت) & مدت بارش) & مقدار بارش & $\begin{array}{c}\text { تاريخ وقوع رَّبار } \\
\text { (روزيار }\end{array}$ \\
\hline$\cdot / 4 \Delta \Delta$ & $\cdot|\cdot r|$ & $K / T$ & • & $T / T^{\varepsilon}$ & $1 \% \wedge 9 / \cdot / T$. \\
\hline.$/ 49$ &.$\cdots \cdot r$ & $r / V$ & $1 / 90$ & $F / 1$ & $1 \% \wedge q / \cdot 1 / r q$ \\
\hline$\cdot / \& \mathrm{~V} \Lambda$ & $\cdot|\cdots r|$ & $1 / 4$ & Q/99 & r. & $\mid r \wedge q / \cdot \% / F$ \\
\hline $11 / \pi 9$ & $.1 . .1$ & $r / 4$ & $9 / 4$ & 1.11 & $\mid r \wedge q / r / 1 r$ \\
\hline F/Te & $.1 . .1$ & ג/ & 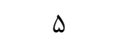 & GIV & 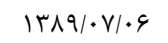 \\
\hline . NYFF & $\cdot \cdots r$ & r & G/QT & v & $\mid r \wedge 9 / \cdot \Lambda \cdot 9$ \\
\hline 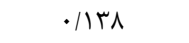 & $\cdot 1 \ldots 19$ & Q/9 & - /Nr & $r / \Lambda$ & $\mid r \wedge q / \wedge / 1 r$ \\
\hline $1 / \omega$ & $\cdot \cdots r V$ &.$/ 49$ &.$/ . \Lambda r$ &.$/ . r$ & $1 r q . / . r / \mid r$ \\
\hline$r \Delta T / \& \varphi$ & $\cdot|\cdot+4|$ & 19 & .199 & iQ & $|r q \cdot / \cdot r / r|$ \\
\hline$r / 99$ & $\cdot \cdots \Delta r$ & $9 / \pi$ & $1 / \pi \Lambda$ & F/9 & $\mid r q \cdot / \cdot r / \cdot r$ \\
\hline ז/M & . & $1 / \pi$ & $\cdot / \cdot \lambda r$ & $\cdot / \mathrm{V}$ & $\mid r q \cdot / \cdot \Delta / \cdot 1$ \\
\hline$r \cdot / 4 q$ & $.1 \ldots \varphi$ & $\Delta / r$ & 年 & r/9 & $1 \% q \cdot 1 \cdot 0 / 19$ \\
\hline $1 . / 91 \mathrm{r}$ & $\cdot 1 . .1$ & $1 / \mathrm{V}$ & $\cdot \pi$ & .19 & $1 r q .1 .91 . r$ \\
\hline $9 / \pi 9$ & $\cdot|\cdot| f$ & $r$ & $1 / r \lambda r$ & $1 / 9$ & $1 \mathrm{rq} .1 .91 . \mathrm{V}$ \\
\hline T/TY & 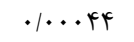 & $V / T$ & $\cdot|\Delta|$ & $r / \Lambda$ & $14 q \cdot 1 \cdot v 1 \cdot q$ \\
\hline
\end{tabular}

تغيير احتمالى آن در كنترل تخمين مدلهاى مدرو مورد

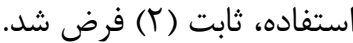

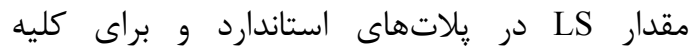

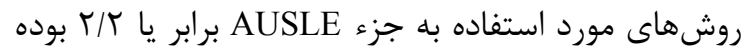

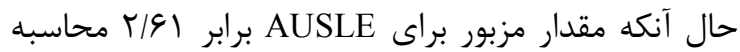
شد.

مقدار C نظر به عدم تغيير :وشش زياهى طى دوره

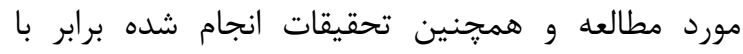

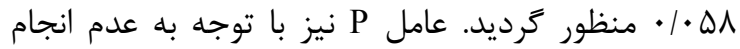

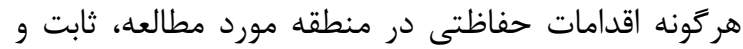

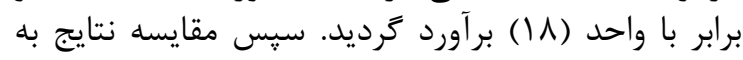

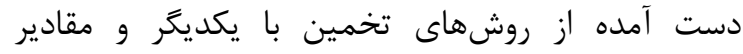

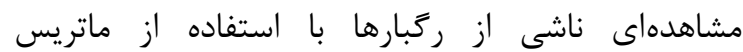

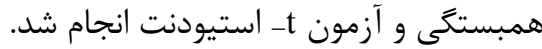

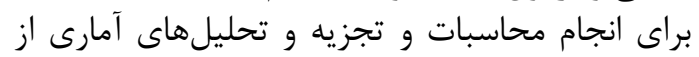

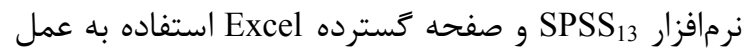

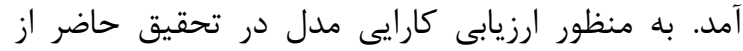

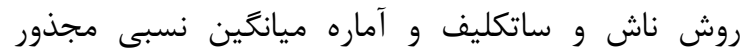

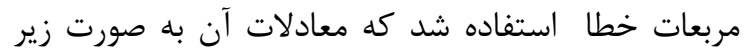

\section{$\mathbf{E}=\frac{\sum_{\mathrm{t}=1}^{\mathrm{n}}\left(\mathbf{Q}_{0}^{\mathrm{t}}-\mathbf{Q}_{\mathrm{s}}^{\mathrm{t}}\right)}{\sum_{\mathrm{t}=1}^{\mathrm{n}}\left(\mathbf{Q}_{0}^{\mathrm{t}}-\mathbf{Q}_{0}^{-}\right)}$}

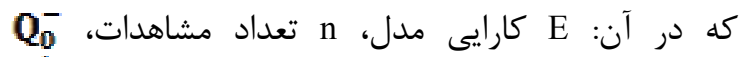
ميانخين مقادير مشاهدهاى،

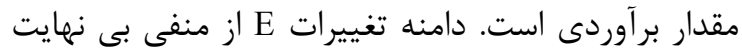

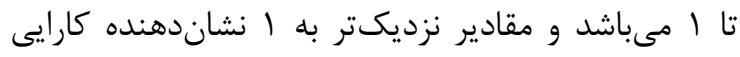

معالات و روابط هر يك از مدلهاى مورد استفاده براى

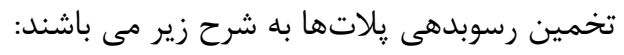
A = R.K.L.S.C.P RMUSLE-E $=1.586\left(\mathrm{Q} . \mathrm{q}_{\mathrm{p}}\right)^{0.56}(\mathrm{DA})^{0.12} \quad$ (T) رابطه (T)

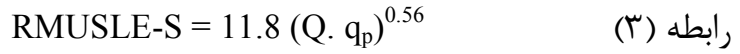
R MUSLT=2.5(Q. qp) LSAUSLE $=(\mathrm{AS} / 22.13)^{0.4} *(\mathrm{Sina} / 0.0896)^{1.3}$ رابطه (ل) در روابط فوق A ميزان فرسايش خاك متوسط سالانه

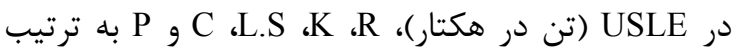

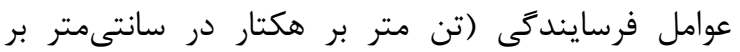

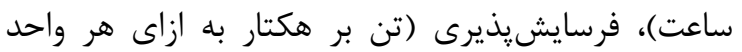

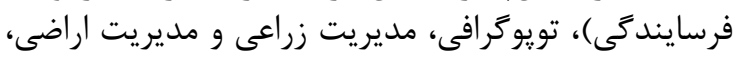

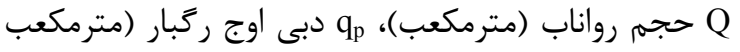

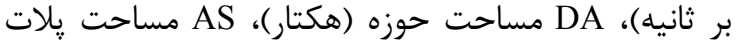

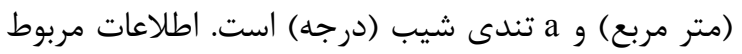

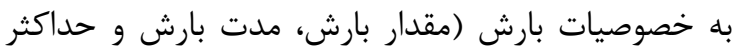

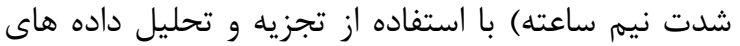
باران نغار استخراج شدند.

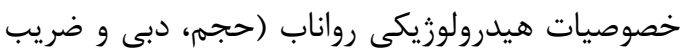

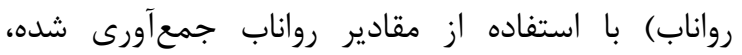

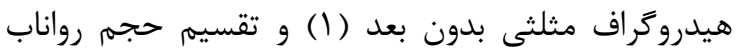

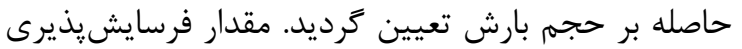

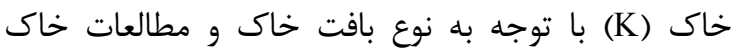

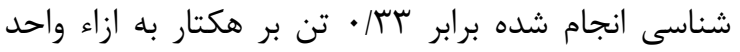

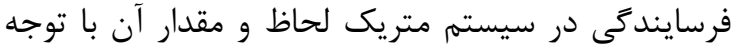

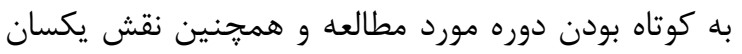




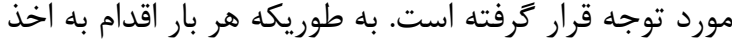

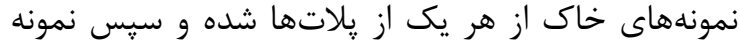

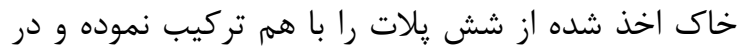

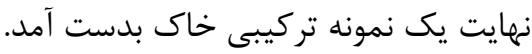

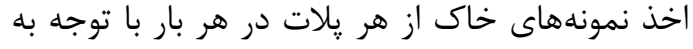

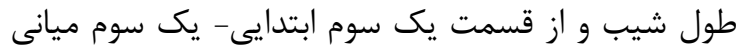

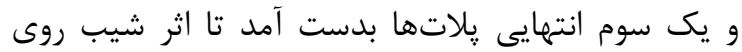

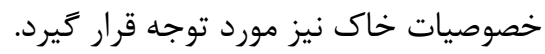

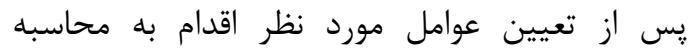

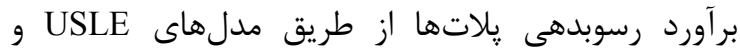

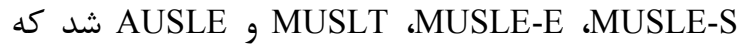

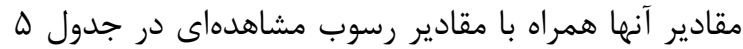

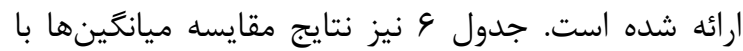

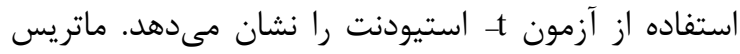

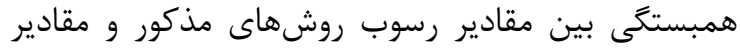

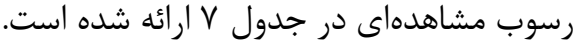

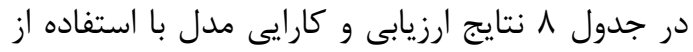

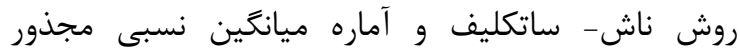
مربعات خطا (RRMSE) نشان داده شده است.
بالاتر مدل و مقاديرى كه به سمت منفى منى بىنهايت ميل مى كند نشاندهنده عدم كفايت مدل هستند

RRMSE $=\frac{\sqrt{\frac{1}{N}} \sum\left(Q_{i}-P_{i}\right)^{2}}{\frac{1}{N} \sum_{i=1}^{N} Q_{i}}$

RRMSE يا ميانگين نسبى مجذور مربعات خطا كه در آن

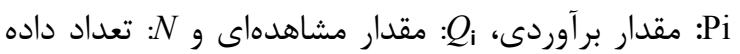

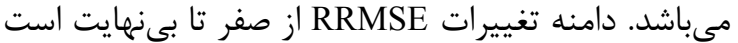
و مقادير نزديكتر به صفر نشاندهنده كارايى بيشتر التئر مدل

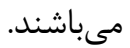

\section{نتايج و بحث}

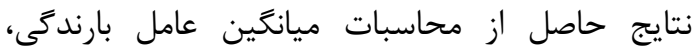

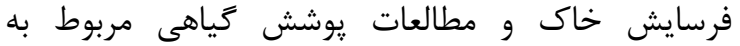

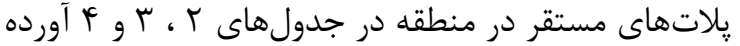

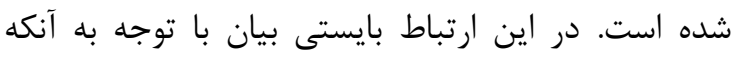

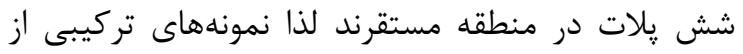

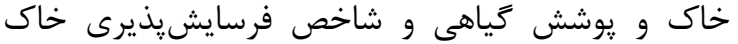

\begin{tabular}{|c|c|c|}
\hline $\begin{array}{c}\text { تاريخ وقوع رَبار } \\
\text { (روز }\end{array}$ & (تن متر بر هكتار بر سانتىمتر) & $\begin{array}{l}\text { عامل بارندىى } \\
\text { (R) }\end{array}$ \\
\hline $1 \% \wedge 9 / \cdot 1 / r \cdot$ & $195 / 19$ & .191 \\
\hline $1 \% \wedge q / \cdot 1 / r q$ & $|\Delta \varepsilon / F|$ & $\cdot \mid \Delta \Lambda$ \\
\hline $\mid r \wedge q / \cdot r / \cdot r$ & r/r & .119 \\
\hline $1 r \wedge q / \cdot r / 1 r$ & IrN/q & $\cdot / A V$ \\
\hline $\mid r \wedge q / \cdot V / \cdot G$ & 1 ITT/G & $\cdot 10$ \\
\hline $\mid r \wedge 9 / \cdot \Lambda / \cdot 4$ & ITF & $\cdot \pi v$ \\
\hline$|r \wedge q / \cdot \Lambda /| r$ & IVT/KG & $\cdot / 9 V$ \\
\hline $1 r q \cdot / \cdot r / 1 r$ & $1 V \cdot / 1$ & $\cdot / \cdot \vee \wedge$ \\
\hline$|r q \cdot| \cdot r / r \mid$ & $r \Lambda F / \Delta$ & $\varphi / \Delta Q$ \\
\hline $\mid r q \cdot / \cdot r / \cdot r$ & $19 \mathrm{~V} / \mathrm{A}$ & $1 / D F$ \\
\hline $1 r q \cdot / \cdot \Delta / \cdot 1$ & $r \cdot r / V$ & $\cdot / K F$ \\
\hline $14 q \cdot / \cdot \Delta / 19$ & $19 \cdot 10$ &.$/ 99$ \\
\hline $1 r q \cdot 1 \cdot 91 \cdot r$ & IVq/F & $\cdot / \pi$ \\
\hline $\mid r q \cdot 1 \cdot 91 \cdot v$ & 199/0 & $\cdot 10$ \\
\hline $\mid r q \cdot 1 \cdot v / \cdot 4$ & $191 / 95$ & $1 / 4 \pi$ \\
\hline
\end{tabular}

جدول بـ- نتايج حاصل از بررسى خصوصيات خاك و فاكتور فرسايشيذيرى آن در پِلاتهاى مستقر در منطقه

\begin{tabular}{|c|c|c|c|c|c|c|c|c|c|}
\hline نمونه تركيبى خاك محل يلاتها & شن & بريار ريز شن & سرصد & رس & مواد آلى & بافت خاك & ساختمان خاى كلاس & نفوذيذيرى كلاس & فرسايش يذيرى خاك \\
\hline يك سوم ابتدايى & rq & $9 / 91$ & $\Delta \varphi$ & F & $F / V V$ & سيلتى لومى & $r$ & $r$ & $\cdot / \pi \Lambda$ \\
\hline يك سوم ميانى & FV & $1 \cdot / T F$ & pq & f & $r / \wedge 9$ & ن لنى لومى & r & r & $\cdot / T_{F}$ \\
\hline يك سوم انتهايى & $f$. & F/AT & $\Delta \varphi$ & f & $r / \cdot r$ & سيلتى لومى & r & r & אז/ן \\
\hline
\end{tabular}




\begin{tabular}{|c|c|c|c|c|c|}
\hline شماره يلات & درصد سنگريزه & درصد خاى لخت & درصد لاشيرگ & درصد يوشش گياهى & ضريب C \\
\hline 1 & rV/A & $19 / 0$ & $F / 1$ & $f / / q$ & $\cdot / \cdot 1$ \\
\hline r & س/T & $I V / \Lambda$ & r/D & DI & $\cdot 1 \cdot \Delta$ \\
\hline f & א/א & $10 / \Lambda$ & $\mathrm{r} / \mathrm{V}$ & $f V / r$ & $\cdot 1 \cdot 9$ \\
\hline$\Delta$ & $r \cdot / l$ & $I T / V$ & $r / \Lambda$ & $s F / r$ & . \\
\hline
\end{tabular}

جدول هـ مقادير رسوب تخمينى مدلهاى MUSLE، MUSLT، MUSLE-E ، و MUSLE-S

\begin{tabular}{|c|c|c|c|c|c|c|}
\hline \multicolumn{5}{|c|}{ مقادير رسوب برآوردى حاصل از نسخ مختلف مورد بررسى متر مر مربع) } & \multirow{2}{*}{ (كَرم در مترمربع) } & \multirow{2}{*}{$\begin{array}{l}\text { رتاريخ وقوع (روز) } \\
\text { رقايخ }\end{array}$} \\
\hline AUSLE & MUSLT & MUSLE-E & MUSLE-S & USLE & & \\
\hline $.1 . \mathrm{T}$ & $.1 \ldots \cdot 19$ & $\cdot 1 \cdot \ldots \cdot 1 r$ & $\cdot 1 \cdots v V$ & $.1 .1 \vee \Delta$ & $\cdot / 4 \Delta \Delta \Delta$ & $1 \% \wedge q / \cdot 1 / T \cdot$ \\
\hline.$/ .14$ & $\cdot|\cdot| \cdot \mid f$ & 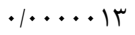 & $\cdot|\cdots \cdot| \wedge$ &.$/ \cdot 1 r$ & .148 & $1 \% \wedge q / \cdot 1 / r q$ \\
\hline$\cdot / \cdot f$ & $\cdot \cdots r \Delta$ & r...... & . $/$..rr & $\cdot / \cdot r$ & $\cdot / 4 V \Lambda$ & $\mid r \wedge q / \cdot r / \cdot r$ \\
\hline . & $\cdot|\cdot| l f$ & $\cdot 1 \cdot \cdots \cdot \mid \Delta$ & . $/$. r r &.$/ \cdot r$ & $11 / 48$ & $1 \% \wedge q / \cdot r / 1 r$ \\
\hline$\cdot / \cdot T \Delta$ & $\cdot 1 \cdot \cdots v r$ & $\cdot / \cdots \cdot \wedge r$ & $\cdot / \cdots \mid r$ & $\cdot 1 \cdot \Delta F$ & $F / T \varphi$ & $\mid r \wedge q / \cdot V / \cdot G$ \\
\hline$\cdot \cdot \cdot v \Delta$ & $\cdot 1 \cdot \ldots r r$ & זr...... & $\cdot / \cdots \Delta \Delta$ & .1 .94 & - $/ V K F$ & $\mid r \wedge q / \cdot \Lambda / \cdot \varphi$ \\
\hline .1 .19 & $.1 . \cdots 19$ & $\cdot / \cdots \cdot \mid r$ & $\cdot / \cdots r F$ & .1 .19 & $\cdot / 1 \mu_{\Lambda}$ & $|r \wedge q / \cdot \wedge /| r$ \\
\hline $.1 . .19$ & $\cdot / \cdots \Delta r$ & r...... & | |...|• & $.1 \cdot .19$ & $1 / 0$ & $|r q \cdot / \cdot r /| r$ \\
\hline . /TrA & $\cdot \cdots \Delta r$ & $\cdot 1 \cdot \cdots \cdot 1$ & $\cdot / \cdot \cdot 1 \mu$ & $.119 r$ & TQH/AS & $|r q \cdot / \cdot r / r|$ \\
\hline$\cdot / \cdot V V$ & $\cdot 1 \ldots \cdot g r$ & $\cdot / \ldots \cdot \varphi$ & •.... & .1 .90 & $r / 99$ & $|r q \cdot| \cdot r / \cdot r$ \\
\hline$\cdot / \cdot V$ & $\cdot / \cdots+\wedge$ & $\cdot 1 \cdot \cdots+v$ & $\cdot \cdots \cdot \Delta r$ & .1 .99 & $\Gamma / \Lambda$ & $|r q \cdot| \cdot \Delta / \cdot 1$ \\
\hline.$/ \cdot 1 r$ & $\cdot 1 \cdots \cdot v 1$ & $\cdot 1 \cdot \cdots \cdot v V$ & $\cdot 1 \cdot \cdots 11$ & $\cdot / \cdot 1 r$ & $r \cdot / 4 q$ & $1 \% q \cdot 1 \cdot \Delta / 19$ \\
\hline$\cdot 1 \cdot \cdot 10$ & $. / \cdot \cdot \mid r$ & $\cdot|\cdot \cdots| r$ & $\cdot 1 \cdots 1 \Delta$ & .1 .14 & $1 . / 91 \mathrm{r}$ & $1 \% q .1 .91 . r$ \\
\hline .1 .199 & $\cdot 1 \cdots \cdot V$ & $\cdot \mid \cdots \cdot 19$ & $.1 \cdots 99$ & .1 .19 & $9 / \pi q$ & $\mid r q \cdot 1 \cdot 91 \cdot V$ \\
\hline $.1 \cdot 1 \wedge \Delta$ & $\cdot / \cdots \cdot \Delta V$ & $\cdot / \cdots \cdot \Delta \Delta$ & $\cdot 1 \cdot \cdots \cdot 91$ & $\cdot / \cdot \Delta \Delta$ & T/TY & $\mid r q \cdot / \cdot v / \cdot q$ \\
\hline
\end{tabular}

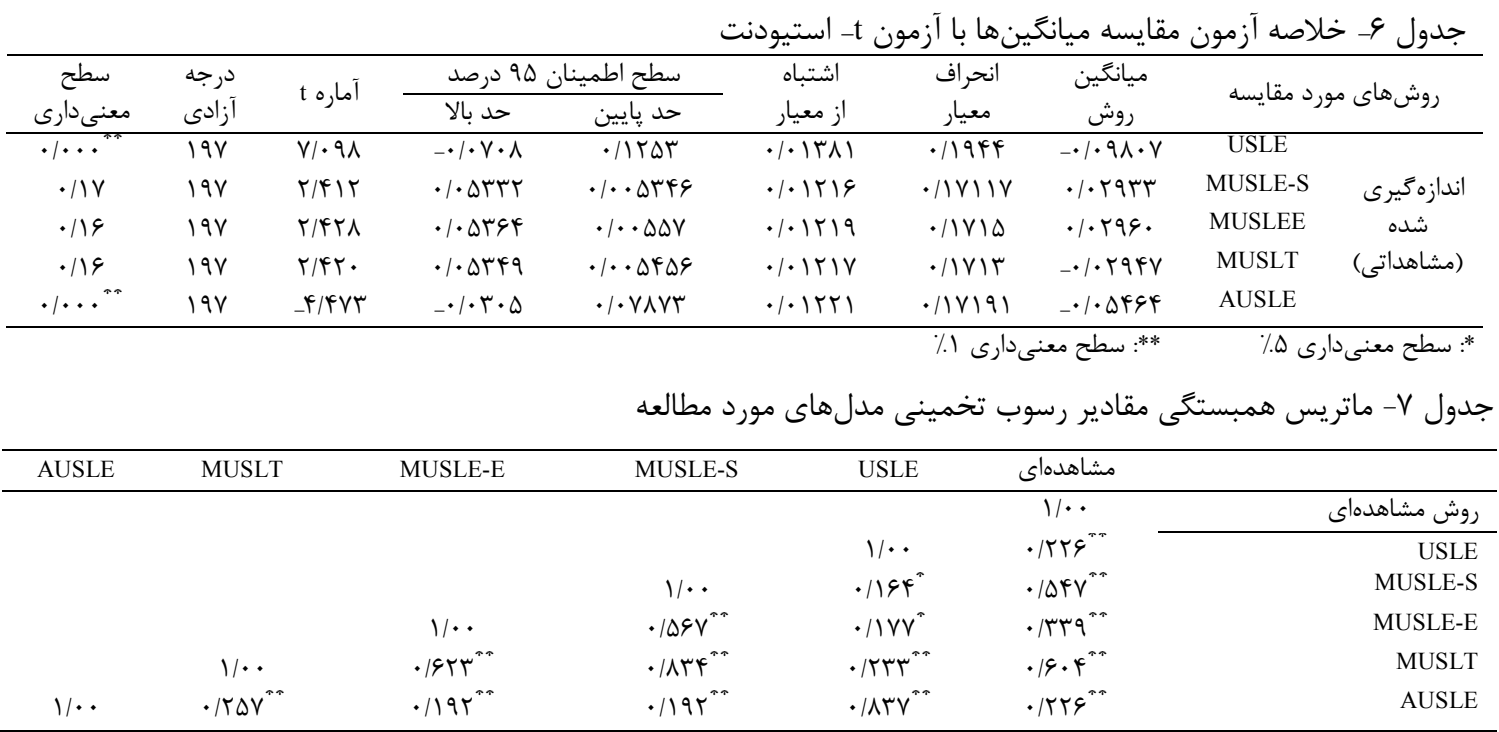

جدول ^ـ اولويت كارايى و رتبهبندى مدلهاى مورد استفاده با استفاده از روش ناش- ساتكليف (ME) و آماره ميانگين نسبى مجذور

\begin{tabular}{|c|c|c|c|c|c|}
\hline & & & & (RRMS & مربعات \\
\hline AUSLE & USLE & MUSLE-S & MUSLE-E & MUSLT & مدل \\
\hline$-\Lambda T r \Delta q$ & $-1.9 V \cdot 9$ & $-r / 1$ & $-\cdot / \Gamma$ & $-\cdot / 1$ & E \\
\hline$T M T / 9$ & $r .$. & $1 / 79$ & 1/9. & $1 / \Delta \Delta$ & RRMSE \\
\hline عدم كارايى & عدم كارايى & سوم & دوم & اول & اولويت كارايى مدل \\
\hline
\end{tabular}


نتايج ارزيابى كارايى مدلها با استفاده از روش ناش- مجات

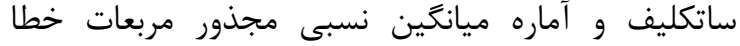

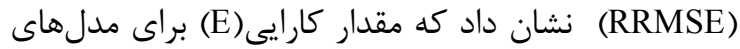
MUSLE-E ،MUSLT

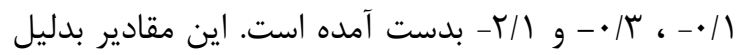

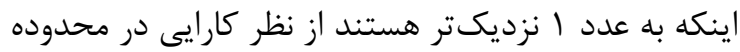

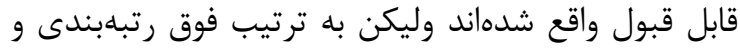

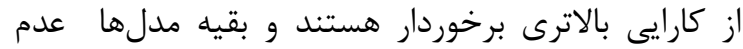
كارايى آنها در تحقيق حاضر مورد تائيد فرار ميى كيردي

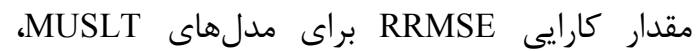
MUSLE-E 1/V9 بدست آمده است اين مقادير بدليل اينكه به عدرد برد

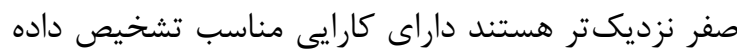

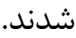

هدف از انجام اين تحقيق ارزيابى كارايى مدل USLE

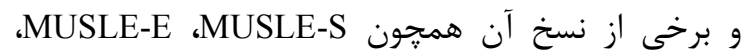

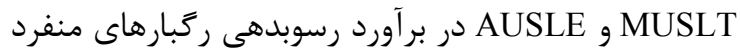

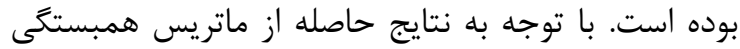

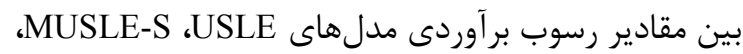
AUSLE و MUSLT ،MUSLE-E

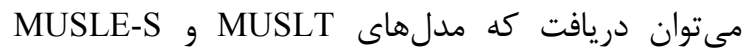

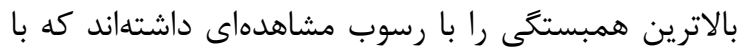

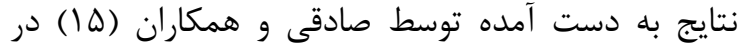

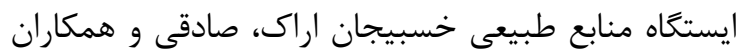

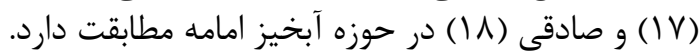

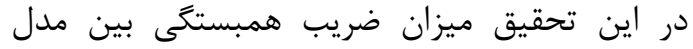

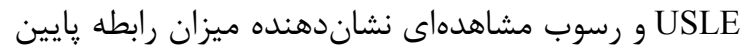

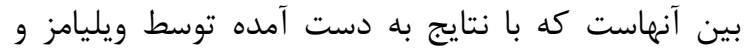

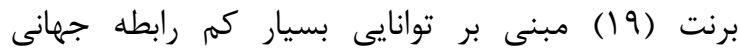

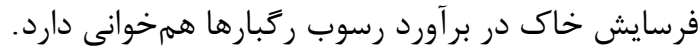

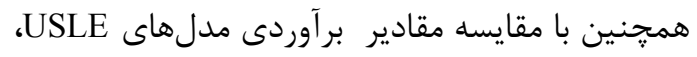

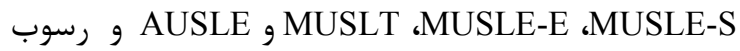
مشاهدهاى با آزمون t- استيودنت مى موان نتيجه كرفت

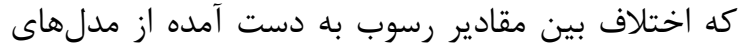

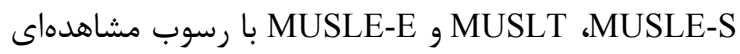

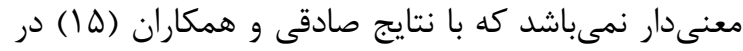

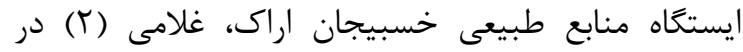

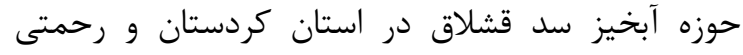

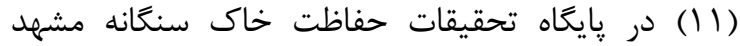

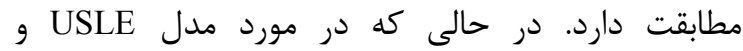
AUSLE

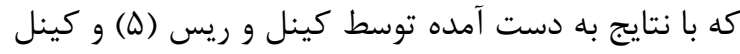

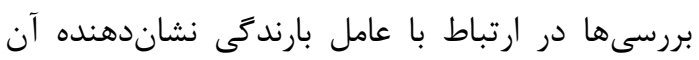

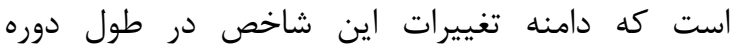

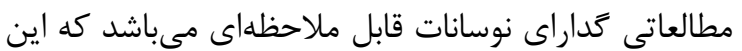

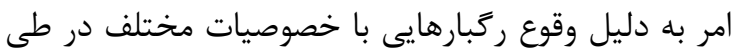

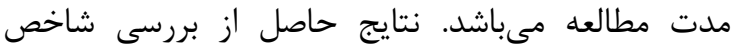

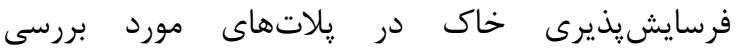

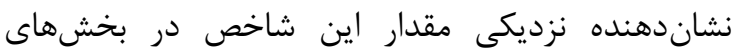

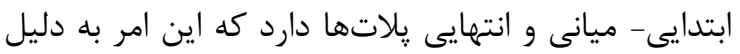

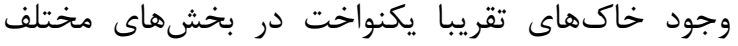
يلاتهاى مستقر مى بـاشد.

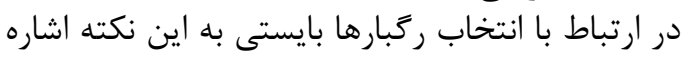

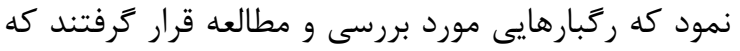

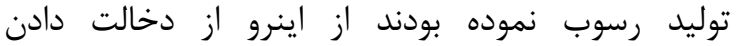

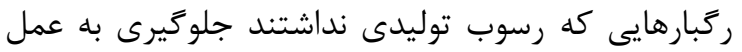

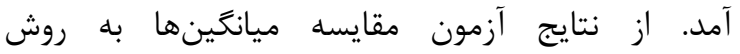

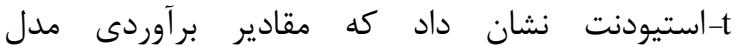
MUSLE-S با سطح معنىدارى بUSLT MUSLE-E

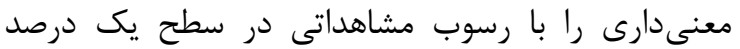

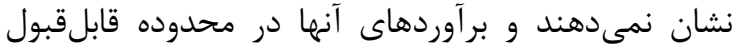

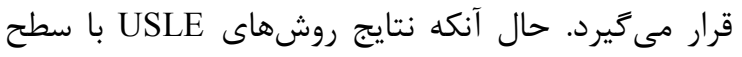

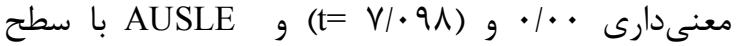

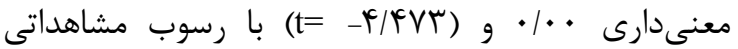

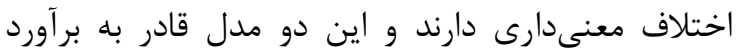

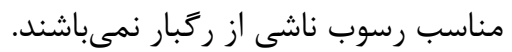

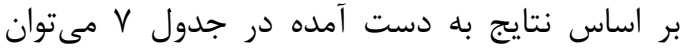

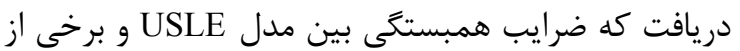

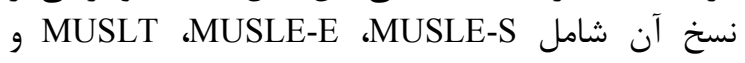
AUSLE و رسوب مشاهدهاى نشان مىدهد كه مدل هاى

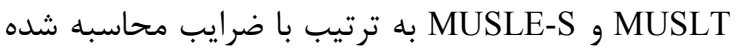

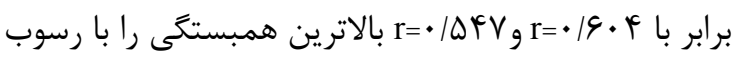

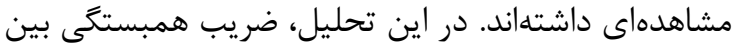

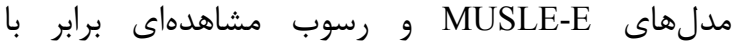

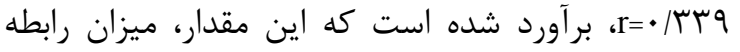

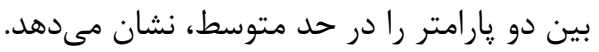

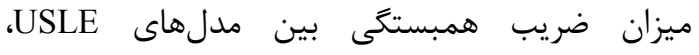

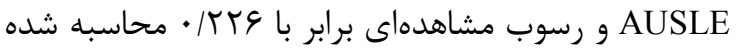

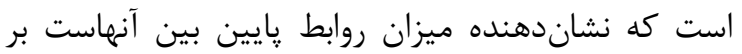

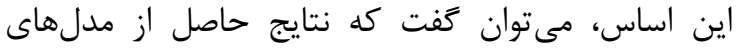

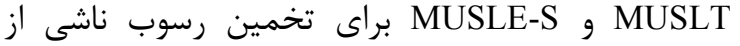

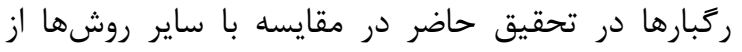
دقت بالاترى برخوردار هستند. 


$$
\begin{aligned}
& r v \cdot \\
& \text { بررسى كارايى رابطه جهانى فرسايش خاك و برخى از نسخ آن در برآورد رسوب رَبارهاى منفرد در . }
\end{aligned}
$$

(Y) در حوزه آبخيز راكى كريك استراليا مبنى بر كارايى

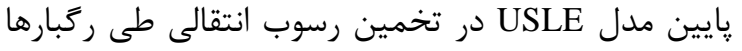

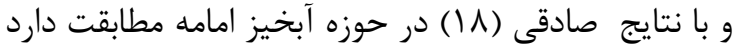

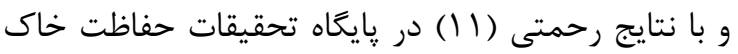

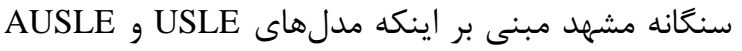
همبستگى معنى دارى با رسوب مشاهداتي دارئى دارد مطابقت ندارد.

منابع

1. Alizade, A. 2004. Practical Hydrology Fundamentals. University of Emam Reza Publications, Thirteen Editions, $570 \mathrm{pp}$ (In Persian).

2. Gholami, L. 2007. Presentation of estimation of sediment production model for Gheshlagh watershed, Kordestan Province M.Sc. Thesis of watershed management Tarbiat Modares University, 135 pp (In Persian).

3. Hashemi, S.A. and M. Arabkhedri. 2008. Evaluation of EPM model by sediment measurement in reservoirs of small dams. Journal of Science and Technology Agriculture and Natural Resources, Water and Soil Sciences, 11: 345-355 (In Persian).

4. Kinnell, P.I.A. 2004. Agriculture non point source pollution model using the USLE-M. AGNPS-UM User, S Guide, University of Canberra, Australia, $210 \mathrm{pp}$.

5. Kinnell, P. I.A. and L.M. Risse. 1998. USLE-M: Empirical modeling rainfall erosion though runoff and sediment concentration. Soil Science Society of American Journal, 62: 1667-1672.

6. Javadi, M.R., F. Mirdar, Sh. Gholami and N. Mashhadi. 2012. Estimation and Comparison of Water Erosion Sedimentation Potential by MPSIAC and EPM Models Using GIS. Journal of Sciences and Techniques in Natural Resources, 7:1-15 (In Persian).

7. Lu, H., C.H. Moran and I. Prosser. 2006. Modelling Sediment Delivery Ratio over the Murray Darling Basin. Environmental Modelling and Software Journal, 21:1297-1308.

8. Olivares, B., K. Verbist, D. Lobo, R.Vargas and O. Silva. 2011. Evaluation of the USLE model to estimate water erosion in an Alfisol. Journal of soil science and plant nutrition, 11: 72-85.

9. Onstad, C.A. and G.R. Foster. 1975. Erosion modeling on a watershed. Trans. Catena, 18: 288-292.

10. Pongsai, S., D.V. Schmidt, P. Rajendra, R. Shestha, S. Clemente and A. Eiumnoh. 2010. Calibration and Validation of the Modified Universal Soil Loss Equation for Estimating Sediment Yield on Sloping Plots: A Case Study in Khun Satan Cachment of northern Thailand.Canadian Journal of Soil Sciences, 90: 585596.

11. Rahmati, S. 2013. Evaluating of Efficiency and Accuracy of USLE and Some of its Versions in Estimating of Event Base Sediment in the Semi-Arid Rangelands. M.Sc. Thesis of watershed management, Islamic Azad University-Nour Branch, 128 pp (In Persian).

12. Rahmati, S., M.R. Javadi and A. Rangavar. 2016. Evaluation of Erosivity factor in USLE Version in Semi-arid Regions of Khorasan. Journal of Environmental Erosion Research, 4:17-29.

13. Refahi, H.Gh. 2000. Soil Erosion by Water and Conservation, Tehran University Press, 485 pp (In Persian).

14. Sadeghi, S.H.R., J.K. Singh and G. Das. 2004. Efficacy of annual soil erosion models for storm-wise sediment prediction. Iran. International Agricultural Engineering Journal, 13: 1-14 (In Persian).

15. Sadeghi, S.H.R., H.R. Pour ghasemi, M. Mohamadi and H. Agha razi. 2008. Assessment of efficiency of USLE and USLE different versions in storm-wise sediment estimation (Khosbijan Natural Resource Research Site-arak). Journal of Agricultural Sciences and Natural Resources, 46: 323-334 (In Persian).

16. Sadeghi, S.H.R. and T. Mizuyama. 2007. Applicability of the modified universal soil loss equation for prediction of sediment yield in Khanmirza watershed, Iran. Hydrological Sciences Journal, 52: 1068-1075.

17. Sadeghi, S.H.R., J.K. Singh and G. Das. 2003. Storm-wise sediment yield prediction using applicable models in Iran. Journal of Agriculture Science and Natural Retours of Khazar, 1: $83-94$ (In Persian).

18. Sadeghi, S.H.R., J.K. Singh and G. Das. 2004. Efficacy of annual soil erosion models for storm-wise sediment prediction. Iran. Agriculture Engineering Journal, 13: 1-14 (In Persian).

19. Williams, J.R. 2010. Sediment-yield prediction with Universal Equation Using Run off Energy Factor. Present and Prospective Technology for Prediction Sediment Yield and Sources, ARS-S-40, US Department of Agriculture, Agricultural Research Service, 25: 244-252.

20. Williams, J.R. and H.D. Berndt. 1977. Sediment yield prediction based on watershed hydrology. Catena, 20: $1100-1104$.

21. Wischmeier, W.H. and D.D. Smith.1978. Predicting rainfall erosion losses-aguide to conservation planning US Department of Agriculture, Agricultural Handbook 537 USDA Washington DC. 238 pp. 


\title{
Efficiency of USLE and some it's Variations for Storm-Wise Sediment Yield in Semi-arid Ranges (Case Study: Margsar Anistitiue of Semnan)
}

\author{
Majid Kargar ${ }^{1}$, Mohammad Reza Javadi ${ }^{2}$, Seyed Ali Asghar Hashemi ${ }^{3}$ and \\ Maryam Mohammad Ebrahimi ${ }^{1}$
1 - M.Sc. Student, Natural Resources Departemant, Islamic Azad University of Noor Branch
2- Assistant Professor, Natural Resources Departemant, Islamic Azad University of Noor Branch (Corresponding author: m_Javadi@iaunour.ac.ir)
3- Scientific Board Member, Natural Resources and Animal Affairs Research Center of Semnan
Received: November 9, 2013 Accepted: April 20, 2015

\begin{abstract}
Severe lack of data on soil erosion and sedimentation in many national watersheds requires the application of appropriate empirical methods for their estimation. This study is aimed to evaluate the efficiencies of Universal Soil Loss Equation (USLE) and some of its variations including MUSLT, MUSLE-E, MUSLE-S and AUSLE in semi-arid climates. This study was undertaken on standard plots in the ranges of Margsar, range and livestock management research station, Jashlobar, Mahdishahr, Semnan province. For this, all of required variables and input data for the respective models have been calculated for the respective plots. Finally, the estimations from both models and measured sediments have been compared during 15 showers. The results of $t$ student's t-test showed that there is no significant difference between MUSLE-E, MUSLT and MUSLE-S models and the measured sediment. Also, the results from efficiencies of the models by NashSutcliffe method as well as relative root mean square error (RRMSE) showed that MUSLE-E, MUSLT and MUSLE-S models have higher efficiencies than other variations.
\end{abstract}

Keywords: USLE, Sedimentation, Semnan province, Standard plots 